\title{
A NOTE ON THE CONNECTIVITY OF COMPONENTS OF KLEINIAN GROUPS
}

\author{
BY \\ CHARLES KALME
}

1. We wish to call attention to an error in a statement on Kleinian groups that says a component of a Kleinian group has connectivity 1, 2, or infinite. This statement appears seemingly for the first time in Appell and Goursat [2, pp. 168169] and subsequently in Lehner [4, Theorem 54, p. 109].

Using a simple construction we will exhibit Kleinian groups with components of arbitrary connectivity (in fact it is possible to construct Kleinian groups with a countable sequence of nonconjugate components, each with a prescribed finite or infinite connectivity), and note under what conditions the original statement does hold. We will observe that the exceptional cases occur only when the subgroup that leaves the component invariant is finite, and we will restrict ourselves to this case. For the sake of completeness we will classify all the possible relations between the connectivity of a component and the order of the subgroup that leaves the component invariant, and finally we will observe that for finitely generated groups the original statement is valid.

The construction used here is standard although the contention that the statement is valid for finitely generated Kleinian groups rests on fairly recent results of Ahlfors [1] and Selberg [5].

2. If $\Gamma$ is a Kleinian group (a properly discontinuous group of Möbius transformations) and $\Delta$ any set in the extended complex plane $\hat{C}$, we denote by $\Gamma_{\Delta}$ the subgroup of $\Gamma$ which leaves $\Delta$ invariant. Two sets $\Delta_{1}$ and $\Delta_{2}$ are said to be congruent modulo $\Gamma$ if there is an element $T \in \Gamma$ such that $\Delta_{2}=T\left(\Delta_{1}\right)$. Congruence modulo $\Gamma$ is an equivalence relation and if $\Delta_{1}$ and $\Delta_{2}$ are congruent, the corresponding groups $\Gamma_{\Delta_{1}}$ and $\Gamma_{\Delta_{2}}$ are conjugate in $\Gamma$, with $\Gamma_{\Delta_{2}}=T \circ \Gamma_{\Delta_{1}} \circ T^{-1}$.

If $D$ is a component of $\Gamma$ (i.e. a component of the region of discontinuity of $\Gamma$ ) and $\Gamma_{D}$ contains loxodromic (including hyperbolic) or parabolic elements, then indeed the connectivity of $D$ is 1,2 , or infinite. The argument used to prove this $\left[4\right.$, p. 109] shows also that if $\Gamma_{D}$ contains parabolic elements the connectivity of $D$ is either 1 or infinite. Since we are interested here in the cases in which this argument is not valid, we will impose the restriction that $\Gamma_{D}$ contain elliptic elements only and therefore, as will be seen, is a finite group.

To each finite group $G$ of Möbius transformations there are attached integers $s$ and $k_{1} \leqq k_{2} \leqq \cdots \leqq k_{s}$ in the following manner:

R sceived by the editors March 9, 1968. 
If $\Lambda$ is the set of fixed points of $G$ (i.e. $p \in \Lambda$ if and only if there exists $T \in G$, $T \neq 1$, such that $T(p)=p$ ), it is invariant under $G$ and can therefore be partitioned into congruence classes modulo $G$, with the number of fixed points congruent to a given point $p$ being equal to the index of $G_{p}$ in $G$. The integer $s$ is then defined to be the number of such congruence classes, while $k_{v}$ is the number of points in the $v$ th congruence class, the integers so arranged that $k_{1} \leqq k_{2} \leqq \cdots \leqq k_{s}$.

The main result can now be stated as follows:

THEOREM. Let $\Gamma$ be a Kleinian group with a component $D$ of connectivity $m$ such that $\Gamma_{D}$ is a finite group of order $N$. If $s$ and $k_{1}, k_{2}, \ldots, k_{s}$ are the integers attached to $\Gamma_{D}$ in the manner previously described, then either $m$ is infinite or

$$
m=n N+\sum_{v=1}^{s} \varepsilon_{v} k_{v}
$$

with $n$ a nonnegative integer and each $\varepsilon_{v}$ either 0 or 1 .

Furthermore, given any finite group $G$ of Möbius transformations with $N$ elements, and any integer $m$ subject only to the above restrictions, $G$ can be extended to $a$ Kleinian group $\Gamma$ that has a component $D$ of connectivity $m$ such that $\Gamma_{D}=G$.

This completely characterizes the possible relations between $m$ and $N$, and the parameters $s$ and $k_{1}, k_{2}, \ldots, k_{s}$ are readily accessible. In fact the finite groups of Möbius transformations have been completely classified [3, Chapter VI, pp. 123126] and fall into five categories: the cyclic groups and groups arising from considering rigid selfmappings of the various regular polyhedra. The parameters $s$ and $k_{1}, k_{2}, \ldots, k_{s}$ determine the group up to conjugacy within the group of all Möbius transformations.

In the process of this classification it is seen that the Riemann surface $(\hat{C}-\Lambda) / \Gamma_{D}$ (i.e. the Riemann sphere with the fixed points of $\Gamma_{D}$ deleted and points congruent modulo $\Gamma_{D}$ identified) is conformally equivalent to the $s$-times punctured sphere, with each conjugacy class of fixed points contributing a puncture. The surface $(D-\Lambda) / \Gamma_{D}$ is then a subdomain of the $s$-times punctured sphere. It also turns out that $s$ is always either 2 or 3 , being 2 only in the case of a cyclic group. These observations will aid in visualizing the proof of the theorem, although the proof itself does not depend on them.

3. In order to prove the first part of our theorem we first observe that any group of Möbius transformations containing only elliptic elements is conjugate within the group of all Möbius transformations to a group that, when projected stereographically, corresponds to a group of rotations of the Riemann sphere [2, pp. 1516 and 61-62]. Since elements that project to rotations take the form $T(z)=$ $(a z-\bar{c}) /(c z+\bar{a})$ with $|a|^{2}+|c|^{2}=1, \Gamma_{D}$, being discrete, must be finite. Assuming further that $D$ has finite connectivity, it is bounded by $m$ continua and can therefore be mapped conformally by a function $f(z)$ onto a domain bounded by $m$ disjoint circles $C_{1}, C_{2}, \ldots, C_{m}$, with the transformed group $f \circ \Gamma_{D} \circ f^{-1}$ again being a finite 
group of Möbius transformations [see 3, Chapter X, pp. 279-283]. Because the properties we are interested in are invariant under such transformations, it can be assumed at the start that $D$ is a domain bounded by $m$ disjoint circles $C_{1}, C_{2}, \ldots, C_{m}$, and $\Gamma_{D}$, when projected stereographically, corresponds to a group of rotations of the Riemann sphere. $\Gamma_{D}$ then acts as a permutation group on the boundary curves and they can be partitioned into congruence classes modulo $\Gamma_{D}$.

The basic idea of the proof is the following: as was previously noted, the surface $S=(\hat{C}-\Lambda) / \Gamma_{D}$ is conformally the $s$-times punctured sphere, with each congruence class of $\Lambda$ corresponding to a puncture, while $D$ is a subdomain of $S$ bounded by simple closed loops, the projections of the boundary curves of $D$. The curves that project to homotopically trivial curves on $S$ come in groups of $N=\operatorname{order}$ of $\Gamma_{D}$, and contribute to the first term on the right hand side of equation (1). The other curves project to loops enclosing the punctures of $S$ and come in groups equal to the number of fixed points of $\Gamma_{D}$ corresponding to the puncture enclosed. These contribute to the second term in the sum. The restriction of the number of such groups (i.e. $\varepsilon_{v}=0$ or 1 ) comes from the requirement that $D$ be connected.

We now proceed with a formal proof.

Lemma 1. If $T \in \Gamma_{D}, T \neq 1$, leaves a boundary curve $C$ invariant then $\Gamma_{C}$ is the maximal cyclic subgroup of $\Gamma_{D}$ containing $T$.

Proof. On the Riemann sphere the invariant circles of $T$ are precisely the ones cut by planes perpendicular to the axis of rotation of $T$. The lemma then follows from the observation that any such circle determines the axis uniquely and all rotations about this axis leave the circle invariant.

The set of maximal cyclic subgroups of $\Gamma_{D}$ can be partitioned into equivalence classes $\mathscr{G}_{1}, \mathscr{G}_{2}, \ldots, \mathscr{G}_{r}$, using the equivalence relation of conjugacy within $\Gamma_{D}$. Adding to these $\mathscr{G}_{0}$, consisting of the trivial subgroup containing only the identity, we denote the resulting set by $\mathscr{G}=\left\{\mathscr{G}_{0}, \mathscr{G}_{1}, \ldots, \mathscr{G}_{r}\right\}$. Associated to each element $\mathscr{G}_{v} \in \mathscr{G}$ is the integer $l_{v}=$ the index in $\Gamma_{D}$ of any one of the maximal cyclic subgroups in the conjugacy class of $\mathscr{G}_{v}$ (note $l_{0}=N=\operatorname{order}$ of $\Gamma_{D}$ ). The set of partitions into congruence classes modulo $\Gamma_{D}$ of the boundary curves of $D$ is denoted by

$$
\not \supset=\left\{\mathscr{P}_{1}, \mathscr{P}_{2}, \ldots, \mathscr{P}_{t}\right\} .
$$

For any boundary curve $C$, either $\Gamma_{C}=\{1\}$ or $\Gamma_{C}$ is a maximal cyclic subgroup of $\Gamma_{D}$ (Lemma 1). Also for $T, S \in \Gamma_{D}$, the subgroup of $\Gamma_{D}$ that leaves $T(C)$ invariant is $T \circ \Gamma_{C} \circ T^{-1}$, and $T(C)=S(C)$ if and only if $S^{-1} T \in \Gamma_{C}$. Thus the nuimber of boundary curves congruent to a given curve $C$ is equal to the index of $\Gamma_{C}$ in $\Gamma_{D}$.

The above properties enable us to define a mapping $\phi: \mathscr{P} \rightarrow \mathscr{G}$ by the rule that $\phi\left(\mathscr{P}_{i}\right)=\mathscr{G}_{v}$ if and only if $\Gamma_{C} \in \mathscr{G}_{v}$ for all $C \in \mathscr{P}_{i}$. The number of boundary curves in the congruence class $\mathscr{P}_{i}$ is then equal to $l_{v}$. Hence $m$ can be expressed by the equation

$$
m=n N+\sum_{v=1}^{r} \delta_{v} l_{v}
$$


where $n$ and $\delta_{v}$ are the number of elements of $\mathscr{P}$ mapped onto $\mathscr{G}_{0}$ and $\mathscr{G}_{v}$ respectively.

A restriction on the $\delta_{v}$ 's is obtained by noting that since the circles $C_{1}, C_{2}, \ldots, C_{m}$ bound a connected domain, at most two can be perpendicular to the same axis, when projected onto the Riemann sphere. Consider a maximal cyclic subgroup $\left\langle T_{v}\right\rangle \in \mathscr{G}_{v}$. On the Riemann sphere the fixed points of this group are the endpoints of the axis of rotation of $T_{v}$. If they are congruent modulo $\Gamma_{D}$, then as soon as there is one invariant circle of $\left\langle T_{v}\right\rangle$ bounding $D$, there must be a second one congruent to it, since the transformation identifying the two fixed points must be a rotation through an angle $\pi$ that maps the invariant circle onto another one distinct from it. This means that $\delta_{v}$ is restricted to the values 0,1 , or 2 if the two fixed points of $\left\langle T_{v}\right\rangle$ are not congruent, and to 0 or 1 if they are congruent.

To relate this condition to the integers $s$ and $k_{1}, k_{2}, \ldots, k_{s}$ associated with $\Gamma_{D}$, we observe that on the Riemann sphere the fixed points of $\Gamma_{D}$ are the endpoints of the axes of rotation of the elements of $\Gamma_{D}$, and since one endpoint already determines its axis, two transformations that have one fixed point in common have a common axis of rotation and hence a common second fixed point. If then to each fixed point we associate the maximal cyclic subgroup of $\Gamma_{D}$ that keeps it fixed, congruent fixed points will correspond to conjugate cyclic subgroups, and the totality of fixed points associated to a given conjugacy class $\mathscr{G}_{v}$ will fall into congruence classes as follows:

(i) if for any $\left\langle T_{v}\right\rangle \in \mathscr{G}_{v}$ the two fixed points of $T_{v}$ are not congruent, there will be two complete sets of congruent fixed points, each containing $l_{v}$ points, associated with $\mathscr{G}_{v}$.

(ii) if the fixed points of $T_{v}$ are congruent there will be one complete set of $l_{v}$ congruent points associated with $\mathscr{G}_{v}$.

It is now easy to see that equation (2) for $m$, with the indicated restrictions on the $\delta_{v}$ 's, is equivalent to

$$
m=n N+\sum_{v=1}^{s} \varepsilon_{v} k_{v}
$$

with $\varepsilon_{v}$ restricted to the values 0 or 1 . This establishes the first part of our theorem.

4. The construction needed to prove the second part of the theorem is based on the following simple lemma.

LeMMA 2. Let $\Sigma_{v}$ and $\Sigma_{v}^{\prime}$ be a nonempty collection of pairs ( finitely or countably many) of simple, closed, oriented Jordan curves with mutually disjoint interiors (by the interior of an oriented Jordan curve we will always mean the region lying on the left, whether bounded or not), and let $T_{v}$ be Möbius transformations mapping $\Sigma_{v}$ onto $\Sigma_{v}^{\prime}$ and taking the interior of $\Sigma_{v}$ onto the exterior of $\Sigma_{v}^{\prime}$.

Let

$$
\Delta_{v}=\left(\text { int } \Sigma_{v}\right) \cup\left(\text { int } \Sigma_{v}^{\prime}\right) \quad \Delta=\bigcup_{v} \Delta_{v} \text {. }
$$


Suppose $G$ is a group of Möbius transformations satisfying

(a) $T(\Delta) \cap S(\Delta)=\varnothing$ if $T, S \in G, T \neq S$.

(b) $D=\operatorname{ext}\left[\bigcup_{T \in G} T(\Delta)\right]$ is nonempty.

Then if $\Gamma$ is the group generated by $G$ and the $T_{v}$ 's, we may conclude that:

(i) $\Gamma$ is properly discontinuous,

(ii) $\Gamma$ is naturally isomorphic to the free product $G * \prod_{v}^{*}\left\langle T_{v}\right\rangle$,

(iii) $\Gamma_{D}=G$.

Proof. From its definition $D$ is easily seen to be invariant under $G$. Since $T_{v}$ maps the exterior of $\Sigma_{v}$ into the interior of $\Sigma_{v}^{\prime}$ and $T_{v}^{-1}$ maps the exterior of $\Sigma_{v}^{\prime}$ into the interior of $\Sigma_{v}$, and the interiors of $\Sigma_{v}$ and $\Sigma_{v}^{\prime}$ are disjoint, it follows that if $T \in\left\langle T_{v}\right\rangle$ and $T \neq 1$ then $T$ maps the exterior of $\Delta_{v}$ into $\Delta_{v}$. Also since for $\nu \neq \mu, \Delta_{v}$ and $\Delta_{\mu}$ are disjoint, and for $T, S \in G, T \neq S, T(\Delta)$ and $S(\Delta)$ are disjoint, it follows that if $W$ is a word in the elements of $G$ and the $T_{v}$ 's, freely reduced in $G * \prod_{v}^{*}\left\langle T_{v}\right\rangle$, then $W(D)$ is either disjoint from $D$ or a subset of $D$ depending on whether $W$ does or does not contain an element of a $\left\langle T_{v}\right\rangle$. Thus the kernel of the natural homomorphism from $G * \prod_{v}^{*}\left\langle T_{v}\right\rangle$ onto $\Gamma$ consists of the identity element only, and the groups are isomorphic. From the above it is also clear that $\Gamma_{D}=G$.

Since elements of $\Gamma$ that are not elements of $G$ map $D$ outside itself, if $G$ is properly discontinuous at a point of $D$ so is $\Gamma$. Hypothesis (a) implies that $G$ is properly discontinuous at every point of $\Delta$, and since the complement of $D$ is a closed set containing more than one point, and invariant under $G$, it contains the limit set of $G$ (see [3, Theorem 5, p. 43]). Hence $G$ is properly discontinuous at every point of $D$, as is $\Gamma$.

This establishes the lemma.

The second part of our theorem can now be proved as follows:

Let $G$ be a finite group of Möbius transformations, $s$ and $k_{1}, k_{2}, \ldots, k_{s}$ the set of integers associated with $G$ as before, $N=$ the order of $G$, and $m$ any finite integer subject to the restrictions of equation (1). It can be assumed without loss of generality that $G$, when projected stereographically, corresponds to a group of rotations of the Riemann sphere. The construction that follows is easier visualized on the Riemann sphere.

It is easily seen that $m$ oriented circles can be chosen in such a way that:

(i) for those $\nu$ for which $\varepsilon_{v}=1$, each fixed point of $G$ belonging to the $\nu$ th congruence class is enclosed by exactly one of the circles, this circle being invariant under the subgroup having the corresponding fixed point, and the $k_{v}$ circles so obtained being congruent modulo $G$.

(ii) the remaining circles form $n$ complete sets with $N$ circles congruent modulo $G$ in each set, with none of these circles enclosing a fixed point of $G$.

(iii) the totality of $m$ circles are mutually disjoint, each being part of the boundary of the connected domain $D$ exterior to all of them.

$D$ is then invariant under $G$ and we can partition the boundary curves into congruence classes modulo $G$. Selecting one representative from each congruence 
class, we insert a countable collection of pairs of positively oriented circles $\Sigma_{\mu}, \Sigma_{\mu}^{\prime}$ (i.e. the bounded region lying on the left), with mutually disjoint interiors, into each of them in such a way that if $C$ is one of the selected curves then-

(a) if $G_{C}=\{1\}$, the $\Sigma_{\mu}$ 's accumulate at each point of $C$.

(b) if $G_{C}$ is cyclic of order $\nu>1$, the $\Sigma_{\mu}$ 's lie in a triangular sector of angle $2 \pi / \nu$, which forms a fundamental region for $G_{C}$ in the interior of $C$, and accumulate at each point of $C$ bounding this sector. Such a construction is clearly possible (see [3, figure on p. 58]).

We now choose Möbius transformations $T_{\mu}$ that map $\Sigma_{\mu}$ onto $\Sigma_{\mu}^{\prime}$, taking the exterior of $\Sigma_{\mu}$ onto the interior of $\Sigma_{\mu}^{\prime}$, and denote by $\Gamma$ the group generated by $G$ and the $T_{\mu}$ 's. From the construction it is evident that the hypotheses of Lemma 2 are satisfied and thus $\Gamma$ is a Kleinian group with $\Gamma_{D}=G$. Furthermore each of the $m$ bounding circles of $D$ consists entirely of limit points of $\Gamma$ and therefore $D$ is in fact a component of $\Gamma$.

The case of infinite connectivity can be obtained similarly by choosing an infinite collection of $N$ congruent circles for which $\Gamma_{C}=\{1\}$, and filling them out as before.

This concludes the proof of our theorem.

Using constructions similar to the one outlined we can obtain Kleinian groups of various kinds. In particular by properly choosing curves to be filled out by the $\Sigma_{\mu}$ 's it is clear that we can construct Kleinian groups with a countable sequence of nonconjugate components each of a prescribed connectivity.

5. It is to be noted that the type of construction used in the previous section always leads to an infinitely generated group, and in fact for finitely generated groups the statement that the connectivity of a component must be 1,2 , or infinite is valid. It was observed in the beginning that exceptions to this statement can occur only if the group $\Gamma$ has a component $D$ such that $\Gamma_{D}$ contains elliptic elements only. That this cannot happen if $\Gamma$ is finitely generated (except in the trivial case when $\Gamma$ itself is finite) is a consequence of the following observations.

A finitely generated Kleinian group $\Gamma$ always contains a torsion free normal subgroup $H$, of finite index in $\Gamma$, that is itself finitely generated [5, Lemma 8, p. 154]. Being of finite index in $\Gamma, H$ has the same limit set as $\Gamma$ [4, Example 2, p. 109], and therefore the same components. If $D$ were a component of $\Gamma$ with $\Gamma_{D}$ consisting only of elliptic elements, then $H_{D}$ would consist of the identity element only. By a result of Ahlfors [1], $D / H_{D}$ would have to be a surface of finite type, which it clearly will not be unless $\Gamma$ itself is finite.

Note. The results in this paper constitute part of the author's doctoral dissertation prepared under the guidance of Professor Lipman Bers and presented in October 1967 at New York University.

\section{REFERENCES}

1. L. V. Ahlfors, Finitely generated Kleinian groups, Amer. J. Math. 86 (1964), 413-429.

2. P. Appell and E. Goursat, Théorie des fonctions algébriques d'une variable, Tome II: Fonctions automorphes (P. Fatou), Gauthier-Villars, Paris, 1930. 
3. L. Ford, Automorphic functions, 2nd ed., Chelsea, New York, 1951.

4. J. Lehner, Discontinuous groups and automorphic functions, Math. Surveys, No. 8, Amer. Math. Soc., Providence, R. I., 1964.

5. A. Selberg, "On discontinuous groups in higher-dimensional symmetric spaces," in Contributions to function theory, Internat. Colloq. Function Theory, Bombay, 1960, pp. 147-164.

\author{
UNIVERSITY OF CALIFORNIA, \\ Berkeley, California
}

